\title{
Comorbidity of Kawasaki disease and group A streptococcal pleural effusion in a healthy child: a case report
}

This article was published in the following Dove Press journal:

International Journal of General Medicine

22 July 2013

Number of times this article has been viewed

\author{
Ahmed H Alhammadi \\ Mohamed A Hendaus \\ General Pediatrics Section, \\ Department of Pediatrics, Hamad \\ Medical Corporation, Doha, Qatar
}

Background: Kawasaki disease is an acute self-limiting vasculitis that affects children. The most dreaded complication of Kawasaki disease reported in the literature over the years is coronary artery disease, which is considered as the main cause of acquired heart disease. However, pulmonary associations with Kawasaki disease have been overlooked. We present a rare, if not unique, case of Kawasaki disease associated with group A streptococcus pleural effusion in the English language literature. A search of the PubMed database was carried out, using a combination of the terms "Kawasaki disease", "pneumonia", and "group A streptococcus". The majority of studies conducted in children with Kawasaki disease have concentrated on the coronary artery implications. Kawasaki disease is considered a self-limiting illness, but can have detrimental consequences if not diagnosed early. When there is a prolonged inflammatory reaction, with no infectious agent identified or remittent fever unresponsive to antibiotics, Kawasaki disease should be taken into consideration. Elevated V $\beta 2+\mathrm{T}$ cells compared with healthy controls suggest possible involvement of a superantigen in the etiology of Kawasaki disease, so it is wise that the health care provider concentrates not only on the cardiac consequences, but also on pulmonary associations.

Keywords: Kawasaki disease, pneumonia, group A streptococcus

\section{Introduction}

Kawasaki disease, also known as mucocutaneous lymph node syndrome, is an acute self-limiting vasculitis of childhood. ${ }^{1}$ Kawasaki disease surpassed rheumatic fever as the most common cause of acquired heart disease. If left untreated, $15 \%-20 \%$ of patients may develop coronary artery lesions and coronary artery dilatation. ${ }^{2}$ The majority of the literature focuses on the cardiac implications of Kawasaki disease, but little is known about its pulmonary complications or associations. Here we report a rare, if not unique, case of Kawasaki disease associated with group A streptococcus (GAS) pleural effusion and a review of the English language literature.

\section{Case presentation}

A 3-year-old female patient presented with a 5-day history of fever, sore throat, and cough, but no rash. Past medical history was unremarkable, as was family history. Immunizations were up to date for age. On admission to the ward, her vital signs were: temperature $39.5^{\circ} \mathrm{C}$; pulse 130 beats per minute; blood pressure $100 / 70 \mathrm{mmHg}$, respiratory rate 50 per minute; oxygen saturation $90 \%$ on oxygen $2 / \mathrm{L}$ per minute via nasal cannula; weight $13.8 \mathrm{~kg}$ (50th percentile); and length $94 \mathrm{~cm}$ (48th percentile). Her skin had normal texture, with no rashes, hypopigmentation, or hyperpigmentation,
Correspondence: Ahmed H Alhammad General Pediatrics Section, Department of Pediatrics, Hamad Medical Corporation, PO Box 3050, Doha, Qatar Tel +974 44392239

Fax +974 4443 957I

Email aalhammadi@hmc.org.qa 
her capillary refill was around 3 seconds, and the oral mucosa was mildly dry. The patient had decreased air entry and coarse crackles in the right lower and middle zones of the lung. The throat was erythematous and there was no cervical lymphadenopathy. The rest of the physical examination was unremarkable.

Initial laboratory results were: white blood cells $24,000 / \mu \mathrm{L}$ (neutrophils 60.2\%, lymphocytes $35.4 \%$, monocytes $4.3 \%$, basophils $0.1 \%$ ), hemoglobin $10.3 \mathrm{~g} / \mathrm{dL}$, and platelets $206,000 / \mu \mathrm{L}$. C-reactive protein was $100 \mathrm{mg} / \mathrm{L}$, erythrocyte sedimentation rate was $65 \mathrm{~mm} /$ hour, albumin was $18 \mathrm{~g} / \mathrm{L}$, creatinine was $66 \mu \mathrm{mol} / \mathrm{L}$, aspartate aminotransferase was $100 \mathrm{U} / \mathrm{L}$, and alanine aminotransferase was $60 \mathrm{U} / \mathrm{L}$. Serum glucose, blood urea nitrogen, calcium, sodium, chloride, and potassium were normal. Venous blood gas was $\mathrm{pH} 7.2, \mathrm{pCO}_{2}$ $44 \mathrm{mmHg}, \mathrm{pO}_{2} 29 \mathrm{mmHg}$, and $\mathrm{HCO}_{3} 17 \mathrm{meq} / \mathrm{L}$, with a base excess of 11. Anteroposterior chest radiographs showed a right middle and lower lobar consolidation with moderate pleural effusion.

\section{Course of hospitalization}

The patient was started on intravenous normal saline $20 \mathrm{~mL} / \mathrm{kg}$ due to dehydration. She was then transferred to the pediatric intensive care unit due to increased oxygen requirement. A chest tube was inserted and $200 \mathrm{~mL}$ of pus was drained. The pleural fluid culture yielded GAS. The patient was started on intravenous cefuroxime and clindamycin. Nasopharyngeal and throat swabs, blood culture, and a Mantoux skin test were all negative.

On the eighth hospital day, there was no clinical response to antibiotics, and fever persisted, with new onset of abdominal pain, diarrhea, and slight tachycardia at a heart rate of 150 beats per minute.

A repeat blood count showed a significant white cell count of $30,000 / \mu \mathrm{L}$, hemoglobin $7.7 \mathrm{~g} / \mathrm{dL}$, platelets $600,000 / \mu \mathrm{L}$, aspartate aminotransferase $180 \mathrm{U} / \mathrm{L}$, alanine aminotransferase $120 \mathrm{U} / \mathrm{L}$, and albumin $18 \mathrm{~g} / \mathrm{L}$, with a rise in erythrocyte sedimentation rate and $\mathrm{C}$-reactive protein.

The differential diagnosis included bacterial resistance to antibiotics, nonbacterial etiologies such as a virus or aspiration of a foreign body, bronchiolitis obliterans, hypersensitivity pneumonitis, atypical Kawasaki disease, eosinophilic pneumonia, Wegener's granulomatosis, pulmonary sequestration, and cystic adenomatoid malformation.

Since atypical Kawasaki disease was in the differential diagnosis, an echocardiogram was ordered which showed a prominent of left anterior descending artery $(0.26 \mathrm{~cm}, \mathrm{z}$-score 3.2$)$ and left main coronary artery
(0.35 cm, z-score 3.2), with no vegetations or pericardial effusion. Our team decided to give one dose of intravenous immunoglobulin $2 \mathrm{~g} / \mathrm{kg}$, and high-dose aspirin $(80 \mathrm{mg} / \mathrm{kg} /$ day) was started. The fever resolved in 24 hours and the patient's general condition improved, but desquamation developed on the hands and feet. The diagnosis of atypical Kawasaki disease was confirmed.

The patient was discharged home in a stable condition on oral antibiotics for 2 weeks and on an antiplatelet dose of aspirin. She was seen in the outpatient clinic in good condition, and a repeat echocardiogram at 6 weeks was normal.

\section{Discussion}

Kawasaki disease is common in Japan, with an incidence of approximately 112 cases per 100,000 children under the age of 5 years, and there were 4248 cases of Kawasaki disease reported in the United States in $2000 .^{3}$ The culprit in Kawasaki disease is not as yet known, so diagnosis might be a challenge for the physician, given that there are no specific tests. However, superantigens might have a role. ${ }^{2}$ In a recent study, Natividad et al concluded that a superantigen could be the possible cause or trigger for Kawasaki disease. The authors based their decision on the elevated levels of V $\beta 2+\mathrm{T}$ cells found in patients with Kawasaki disease compared with healthy controls. ${ }^{4}$ Another study showed that the inflammation in Kawasaki disease could be due to several infectious agents in genetically susceptible persons. Staphylococcal and streptococcal superantigens have also been reported as possible culprits. ${ }^{5}$

The diagnostic criteria for diagnosis of classic Kawasaki disease include high fever for at least 5 days, in addition to four or more of the following symptoms: polymorphous rash, cervical lymphadenopathy (more than $1.5 \mathrm{~cm}$ ), bilateral nonpurulent conjunctivitis, "strawberry" tongue, swollen hands and feet, erythema of the soles of feet and palms, and red, swollen cracked lips. ${ }^{1}$

Atypical Kawasaki disease is the term used to describe patients with incomplete presentation of the disease and usually constitutes up to $36.2 \%$ of all cases of the disease. Cervical lymphadenopathy and extremity changes are the two most common symptoms not seen in a patient with atypical Kawasaki disease. ${ }^{6}$ Since Kawasaki disease is considered to be a vasculitis, symptoms like cough, abdominal pain, diarrhea, and vomiting might appear around ten days prior to the diagnosis of the disease. ${ }^{7}$

In terms of laboratory studies, inflammatory markers that might aid in the diagnosis of Kawasaki disease include: elevated platelet count, erythrocyte sedimentation rate, $\mathrm{C}$-reactive protein, leukocyte count, aspartate aminotransferase, alanine 
aminotransferase, and a high white cell count in urine. However, albumin, sodium, potassium, total cholesterol, lymphocytes, and hemoglobin might be low. ${ }^{8}$ Patients with atypical Kawasaki disease have been reported to have a low frequency of pyuria, low frequency of hyponatremia, low levels of gamma glutamyl transferase, and low levels of serum alanine aminotransferase compared with patients with classical Kawasaki disease. ${ }^{6}$ Kentsis et al found that high concentrations of two urine proteomes (filamin $\mathrm{C}$ and meprin A) are specific for the diagnosis of Kawasaki disease. ${ }^{9}$

There are few cases of Kawasaki disease associated with pulmonary changes, but they were not associated with GAS. De Maddi et $\mathrm{l}^{10}$ and Uziel et $\mathrm{al}^{11}$ described five patients diagnosed as having atypical Kawasaki disease with unspecified pneumonia. Other authors also describe pulmonary changes not related to GAS. ${ }^{2,12-15}$ In addition, Huang et al reported a case of atypical Kawasaki disease associated with Mycoplasma pneumoniae and Epstein-Barr virus infection. ${ }^{16}$

Prompt initiation of therapy in Kawasaki disease can decrease the risk of coronary artery lesions and dilatation from $20 \%$ to $5 \%$. The initial treatment is a single dose of intravenous immunoglobulin $(2 \mathrm{~g} / \mathrm{kg})$ and high-dose aspirin (80-100 mg/kg/day, divided into four doses). Treatment is more effective if started within ten days of onset of fever, but it is also advisable to initiate it even after ten days. The aspirin dose is usually switched from an anti-inflammatory dose to an antiplatelet dose (3-5 mg/kg/day, given as a single dose) after 48-72 hours of defervescence. The antiplatelet dose of aspirin is usually continued for 6-8 weeks after the child is afebrile and if there are no coronary artery abnormalities. ${ }^{17}$

Retreatment with a second dose of intravenous immunoglobulin $(2 \mathrm{~g} / \mathrm{kg})$ is recommended if there is persistent fever for $\geq 36$ hours after completion of the initial intravenous immunoglobulin infusion. ${ }^{18}$

Steroids have been used for refractory cases of Kawasaki disease, especially the combination of intravenous methylprednisolone and oral corticosteroids; however, their use is controversial because of some reports indicating that steroids might cause coronary artery aneurysm and rupture. ${ }^{19}$

Tumor necrosis factor-alpha might play a role in the inflammatory process in acute Kawasaki disease, so studies were conducted on the efficacy and safety of anti-tumor necrosis factor-alpha (infliximab) for the treatment of refractory cases of Kawasaki disease. Hirono et al reported success using infliximab in eight of 11 patients with refractory Kawasaki disease, with success measured by resolution of fever and a decrease in levels of serum interleukin-6, soluble tumor necrosis factor-alpha receptor 1 , and C-reactive protein. ${ }^{20}$
Burns et al reported a complete response to infliximab therapy, with cessation of fever and decrease in C-reactive protein in 14 of 16 patients. ${ }^{21}$ Song et al also reported a complete response of $80 \%$ in patients with refractory Kawasaki disease after use of infliximab. The response was measured by defervescence and decreased levels of C-reactive protein. ${ }^{19}$

\section{Conclusion}

Kawasaki disease is considered a self-limiting disease, but it can have detrimental consequences if not diagnosed early. When there is a prolonged inflammatory reaction and no infectious agent is identified or there is remittent fever unresponsive to antibiotics, Kawasaki disease should be taken into consideration. The finding of elevated V $\beta 2+\mathrm{T}$ cells suggests possible involvement of a superantigen in the etiology of Kawasaki disease, so it is wise for the health care provider to concentrate not only on the cardiac consequences, but also on pulmonary associations.

\section{Disclosure}

The authors report no conflicts of interest in this work.

\section{References}

1. Coustasse A, Larry J, Lee D. Can Kawasaki disease be managed? Perm J. 2012;16:70-72.

2. Lee MN, Cha JH, Ahn HM, et al. Mycoplasma pneumoniae infection in patients with Kawasaki disease. Korean J Pediatr. 2011;54:123-127.

3. Newburger JW, Takahashi M, Gerber MA, et al; Committee on Rheumatic Fever, Endocarditis, and Kawasaki Disease, Council on Cardiovascular Disease in the Young, American Heart Association. Diagnosis, treatment, and long-term management of Kawasaki disease: a statement for health professionals from the Committee on Rheumatic Fever, Endocarditis, and Kawasaki Disease, Council on Cardiovascular Disease in the Young, American Heart Association. Pediatrics. 2004;114:1708-1733.

4. Natividad MF, Torres-Villanueva CA, Saloma CP. Superantigen involvement and susceptibility factors in Kawasaki disease: profiles of TCR V $\beta 2+\mathrm{T}$ cells and HLA-DRB1, TNF- $\alpha$ and ITPKC genes among Filipino patients. Int J Mol Epidemiol Genet. 2013;4:70-76.

5. Matsubara K, Fukaya T. The role of superantigens of group A Streptococcus and Staphylococcus aureus in Kawasaki disease. Curr Opin Infect Dis. 2007;20:298-303.

6. Yu JJ. Diagnosis of incomplete Kawasaki disease. Korean J Pediatr. 2012;55:83-87.

7. Baker AL, Lu M, Minich LL, et al. Associated symptoms in the ten days before diagnosis of Kawasaki disease. J Pediatr. 2009;154:592-595.

8. Lee KY, Rhim JW, Kang JH. Kawasaki disease: laboratory findings and an immunopathogenesis on the premise of a "protein homeostasis system”. Yonsei Med J. 2012;53:262-275.

9. Kentsis A, Shulman A, Ahmed S, et al. Urine proteomics for discovery of improved diagnostic markers of Kawasaki disease. EMBO Mol Med. 2013;5:210-220.

10. De Maddi F, Cinelli R, Rigante D, Mazzarella G, Siani P. Lung parenchymal consolidation as an uncommon presentation and cause of delayed diagnosis in atypical Kawasaki syndrome. Rheumatol Int. 2009;29:1373-1376. 
11. Uziel Y, Hashkes PJ, Kassem E, Gottesman G, Wolach B. "Unresolving pneumonia" as the main manifestation of atypical Kawasaki disease. Arch Dis Child. 2003;88:940-942.

12. Voynow JA, Schanberg L, Sporn T, Kredich D. Pulmonary complications associated with Kawasaki disease. J Pediatr. 2002;140:786-787.

13. Freeman AF, Crawford SE, Finn LS, et al. Inflammatory pulmonary nodules in Kawasaki disease. Pediatr Pulmonol. 2003;36:102-106.

14. Kobayashi Y, Koike Y, Tokutomi T, Kuroki Y, Todoroki I. Case 2: fever, rash and pulmonary involvement. Diagnosis: Kawasaki disease. Acta Paediatr. 2006;95:1145-1148.

15. Sengler C, Gaedicke G, Wahn U, Keitzer R. Pulmonary symptoms in Kawasaki disease. Pediatr Infect Dis J. 2004;23:782-784.

16. Huang FL, Chang TK, Jan SL, et al. Co-morbidity of Kawasaki disease. Indian J Pediatr. 2012;79:815-817.
17. Freeman AF, Shulman ST. Kawasaki disease: summary of the American Heart Association guidelines. Am Fam Physician. 2006;74: 1141-1148.

18. Kuo HC, Yang KD, Chang WC, Ger LP, Hsieh KS. Kawasaki disease: an update on diagnosis and treatment. Pediatr Neonatol. 2012;53:4-11.

19. Song MS, Lee SB, Sohn S, et al. Infliximab treatment for refractory Kawasaki disease in Korean children. Korean Circ J. 2010;40: 334-338.

20. Hirono K, Kemmotsu Y, Wittkowski H, et al. Infliximab reduces the cytokine-mediated inflammation but does not suppress cellular infiltration of the vessel wall in refractory Kawasaki disease. Pediatr Res. 2009;65:696-701.

21. Burns JC, Mason WH, Hauger SB, et al. Infliximab treatment for refractory Kawasaki syndrome. J Pediatr. 2005;146:662-667.

\section{Publish your work in this journal}

The International Journal of General Medicine is an international, peer-reviewed open-access journal that focuses on general and internal medicine, pathogenesis, epidemiology, diagnosis, monitoring and treatment protocols. The journal is characterized by the rapid reporting of reviews, original research and clinical studies across all disease areas.
A key focus is the elucidation of disease processes and management protocols resulting in improved outcomes for the patient.The manuscript management system is completely online and includes a very quick and fair peer-review system. Visit http://www.dovepress.com/ testimonials.php to read real quotes from published authors. 